\title{
Distinct Roles of SNARE-mimicking Lipopeptides during Initial Steps of Membrane Fusion
}

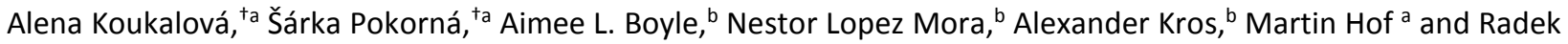 \\ Šachl *a \\ aDepartment of Biophysical Chemistry, J. Heyrovský Institute of Physical Chemistry of the Academy of Sciences of the Czech Republic, Prague, 182 \\ 23, Czech Republic, E-mail: radek.sachl@jh-inst.cas.cz,

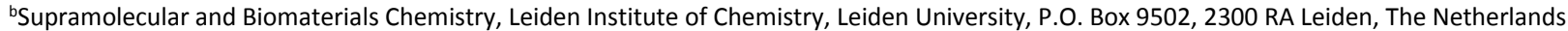 \\ †AK:á and ŠP contributed equally to this work.
}

\begin{abstract}
A model system for membrane fusion, inspired by SNARE proteins and based on two complementary lipopeptides $\mathrm{CP}_{n} \mathrm{E}_{4}$ and $\mathrm{CP}_{n} \mathrm{~K}_{4}$, has been recently developed. It consists of cholesterol (C), a poly(ethylene glycol) linker ( $\left.\mathrm{P}_{n}\right)$ and either a cationic peptide $\mathrm{K}_{4}(\mathrm{KIAALKE})_{4}$ or an anionic peptide $\mathrm{E}_{4}$ (EIAALEK) 4 . In this paper, fluorescence spectroscopy is used to decipher distinct but complementary roles of these lipopeptides during early stages of membrane fusion. Molecular evidence is provided that different distances of $\mathrm{E}_{4}$ in $\mathrm{CP}_{n} \mathrm{E}_{4}$ and $\mathrm{K}_{4}$ in $\mathrm{CP}_{n} \mathrm{~K}_{4}$ from the bilayer represent an important mechanism, which enables fusion. Whereas $E_{4}$ is exposed to the bulk and solely promotes membrane binding of $\mathrm{CP}_{n} \mathrm{~K}_{4}, \mathrm{~K}_{4}$ loops back to the lipid-water interface where it fulfills two distinct roles: it initiates bilayer contact by binding to $\mathrm{CP}_{n} \mathrm{E}_{4}$ containing bilayers; and it initiates fusion by modulating the bilayer properties. The interaction between $\mathrm{CP}_{n} \mathrm{E}_{4}$ and $\mathrm{CP}_{n} \mathrm{~K}_{4}$ is severely down-regulated by binding of $\mathrm{K}_{4}$ to the bilayer and possible only if the lipopeptides approach each other as constituents of different bilayers. When the complementary lipopeptides are localized in the same bilayer, hetero-coiling is disabled. These data provide crucial insights as to how fusion is initiated and highlight the importance of both peptides in this process.
\end{abstract}

\section{Keywords}

membrane fusion, lipopeptides, SNARE, FRET, FCS

\section{Introduction}

Fusion of cellular membranes has recently attracted considerable scientific attention, not only for being ubiquitous and vital in living organisms, but also for its potential to be used for in vivo applications. Fusion of membranes in living cells is crucial for a number of cellular functions, e.g. the controlled release of neurotransmitters, fertilization, communication, and material exchange in eukaryotic cells. ${ }^{1,2}$ In eukaryotic cells, this process is mediated by so-called SNARE proteins (soluble $\mathrm{N}$-ethyl-maleimide-sensitive factor attachment protein receptor), which have been proposed to be involved in all intracellular events of membrane fusion. ${ }^{3}$ The fusing membranes are brought into contact by the formation of a tetrameric coiled-coil between three different membrane-tethered SNARE proteins. This so-called coiled-coil interaction is established by $\alpha$-helical portions of the proteins interacting with each other in a specific manner resulting in the formation of a stable complex. ${ }^{4,5}$

Despite the huge diversity of systems where fusion can occur, the fusion cascade shares a few common features: first, contact between two membranes is developed and accompanied by disruption of the contact site. This is followed by fusion of the proximal leaflets and lipid mixing, which culminates in opening of a fusion pore and content mixing. ${ }^{1,6,7}$ Attempts to mimic and understand the mechanism of membrane fusion has led to the development of several artificial model systems using various strategies, e.g. double stranded DNAs, covalent or hydrogen-bonding motifs and coiled-coil interactions between two complementary $\alpha$-helices. ${ }^{8-11}$ Recently, a model system inspired by the molecular recognition of native SNARE proteins has been developed. ${ }^{12}$ The fusogens consist of a set of two 
complementary lipopeptide molecules (Figure 1). Each of these lipopeptides contains a recognition domain comprising one of the complementary coiled-coil-forming peptides $\mathrm{E}$ or $\mathrm{K}$, cholesterol which serves as a membrane anchor and a poly(ethylene glycol) (P) of variable length. The latter molecule serves as a linker between the cholesterol anchor and the peptide. If the cationic peptide $\mathrm{K}_{4}(\mathrm{KIAALKE})_{4}$ is employed, the construct is known as $\mathrm{CP}_{n} \mathrm{~K}_{4}$, where $n$ denotes the number of ethylene glycol units, and $\mathrm{CP}_{n} \mathrm{E}_{4}$ represents the construct containing the anionic peptide $\mathrm{E}_{4}(\mathrm{EIAALEK})_{4}$. The lipopeptides $\mathrm{CP}_{n} \mathrm{~K}_{4}$ and $\mathrm{CP}_{n} \mathrm{E}_{4}$ interact with each other by the formation of a coiledcoil motif resulting from the interaction of peptide $\mathrm{E}_{4}$ and $\mathrm{K}_{4}$. This coiled-coil is strong enough to bring the two opposing membranes into close contact and induce effective and leakage-free fusion in vitro and in vivo. ${ }^{12,13}$ Both lipopeptides can be incorporated in artificial as well as plasma membranes of living cells in a facile manner by adding the lipopeptides directly to either liposomes or cells. This strategy opens up new possibilities for in vivo applications. ${ }^{13,14,15}$ However, the exact mechanism of lipopeptide-mediated membrane fusion still remains unknown. In this paper, we employ FCS (fluorescence correlation spectroscopy) and FCCS (fluorescence crosscorrelation spectroscopy) techniques and FRET (Förster resonance energy transfer) to uncover distinct but complementary roles of $\mathrm{CPn}_{4}$ and $\mathrm{CP}_{n} \mathrm{~K}_{4}$ lipopeptides during the initial steps of membrane fusion. We show for the first time that the interaction between the complementary lipopeptides, which is a pre-requisite for establishing a membrane contact between two approaching bilayers, is strongly down-regulated by the looping-back of $\mathrm{K}_{4}$ to the lipid bilayer. The majority of $\mathrm{CP}_{n} \mathrm{~K}_{4}$ is in fact needed to poise the bilayer for undergoing fusion whereas only a minor fraction of $\mathrm{CP}_{n} \mathrm{~K}_{4}$ is then used to bind $\mathrm{CP}_{n} \mathrm{E}_{4}$ on an approaching bilayer. Conversely, $\mathrm{E}_{4}$ in $\mathrm{CP}_{n} \mathrm{E}_{4}$ is largely exposed to the bulk and works solely as a handle for $\mathrm{CP}_{n} \mathrm{~K}_{4}$. This paper provides molecular evidence that different distances, facilitated by different PEG-spacer lengths, of $\mathrm{E}_{4}$ in $\mathrm{CP}_{n} \mathrm{E}_{4}$ and $\mathrm{K}_{4}$ in $\mathrm{CP}_{n} \mathrm{~K}_{4}$ from the lipid-water interface represent an important mechanism which enables efficient fusion.

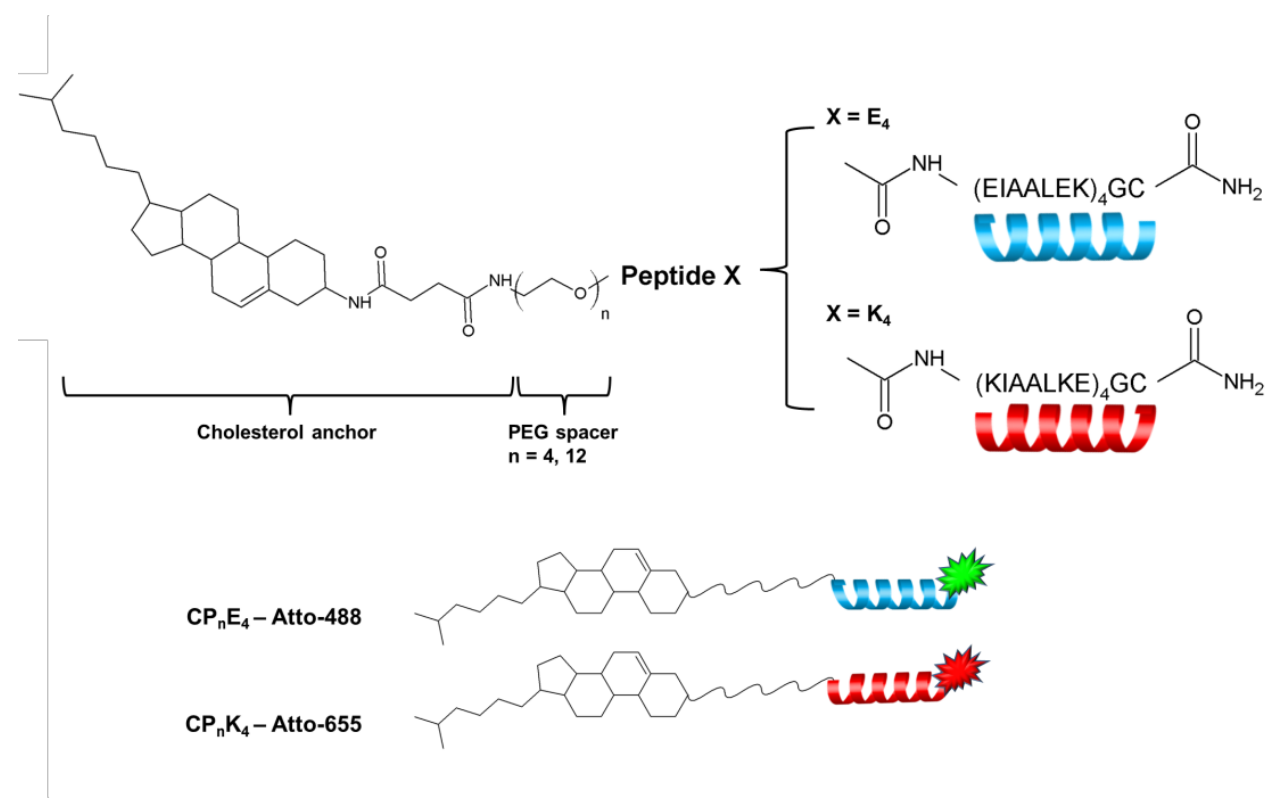

Figure 1. Chemical structures of $\mathrm{CP}_{n} \mathrm{E}_{4}$ and $\mathrm{CP}_{n} \mathrm{~K}_{4}$ lipopeptides. A lipopeptide consists of a cholesterol moiety (C), a flexible poly(ethylene glycol) (PEG) linker of either 4 or 12 PEG units ( $P_{4}$ or $\left.P_{12}\right)$ and one of the complementary peptides $\mathrm{E}$ or $\mathrm{K}$. The lipopeptides were fluorescently labelled with either Atto-488 or Atto-655 at the $\mathrm{C}$ terminus of the peptide by a thiol-maleimide coupling.

\section{Results and Discussion}

\section{Strong binding of $\mathrm{K}_{4}$ to the bilayer hampers a direct interaction of $\mathrm{E}_{4}$ with $\mathrm{K}_{4}$}


In alignment with previous studies, we used 1,2-dioleoyl-sn-glycero-3-phosphocholine/1,2-dioleoyl-snglycero-3-phosphoethanolamine/Cholesterol (DOPC/DOPE/Chol) (50/25/25 mol\%) lipid mixtures as fusion of such DOPE rich membranes was found to be highly efficient due to induction of negative curvature by the DOPE lipids. ${ }^{16-19}$ The extent of membrane binding was determined by measuring the fluorescence intensity of the peptides bound to the bilayer of giant unilamellar vesicles (GUVs) and confirmed by z-scan FCS diffusion measurements (see Supporting Information). The GUVs incubated solely with a fluorescent peptide $\mathrm{K}_{4}$-Atto-655 exhibited approximately 6.5 times higher intensity than those incubated with the same concentration of $\mathrm{E}_{4}$-Atto-655, showing a higher affinity of $\mathrm{K}_{4}$ to the lipid bilayer (Figure 2). The situation changed drastically when the vesicles were decorated with one of the complementary lipopeptides prior to addition of either $E_{4}-A t t o-655$ or $K_{4}$-Atto-655 (Figure 2). Binding of both $E_{4}$-Atto-655 and $K_{4}$-Atto-655 significantly improved, which demonstrates the mutual affinity of the complementary peptides.

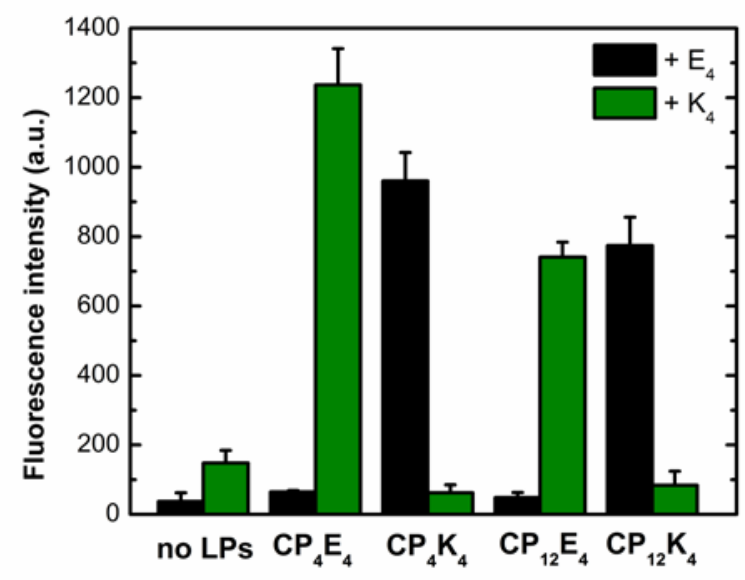

Figure 2. Fluorescence intensities (averaged number of photons per area) of fluorescently labelled peptides $\mathrm{K}_{4}$-Atto-655 (olive) and E4-Atto-655 (black) adsorbed on the surface of DOPC/DOPE/Chol (50/25/25 mol\%) GUVs give information about the surface concentration of the peptides at the lipid bilayer. The GUVs were prepared either with or without $1 \mathrm{~mol} \%$ of one of the complementary lipopeptides (LPs) (type of the lipopeptide is further specified below the $x$ axis). GUVs were scanned 30 minutes after the addition of the peptide $(0.4 \mathrm{~mol} \%)$.

To understand how binding of the peptides to the complementary lipopeptides progresses at the molecular level, fluorescence correlation spectroscopy (FCS) experiments were conducted. An output of an FCS measurement is an autocorrelation function ( $\left.G_{\text {auto }}\right)$, which contains information about diffusion of fluorescently labelled molecules (Figure 3A). The diffusion coefficient $D$ of $K_{4}$-Atto-655 in bilayers with and without $1 \mathrm{~mol} \%$ of $\mathrm{CP}_{4} \mathrm{E}_{4}$ remained almost the same and was similar to the diffusion coefficient of fluorescently labelled DOPE-Atto-655 lipids in the presence of $1 \mathrm{~mol} \%$ of $\mathrm{K}_{4}$ (Table 1). Moreover, the diffusion of $\mathrm{K}_{4}$-Atto- 655 in the presence of 1 mol\% of $\mathrm{CP}_{4} \mathrm{E}_{4}$ was slightly higher than the diffusion of $\mathrm{CP}_{4} \mathrm{E}_{4}-$ Atto-488 in the presence of $1 \mathrm{~mol} \%$ of $\mathrm{K}_{4}$. The last two observations would indicate that a considerable fraction of $\mathrm{K}_{4}$-Atto- 655 stays in the bilayer unbound to $\mathrm{CP}_{4} \mathrm{E}_{4}$-Atto-488. The amount of unbound $\mathrm{K}_{4}-\mathrm{Atto}-655$ was further quantified by fluorescence cross-correlation spectroscopy (FCCS) experiments between $\mathrm{K}_{4}$-Atto655 and $\mathrm{CP}_{4} \mathrm{E}_{4}$-Atto-488 (see SI for further details). In Figure 3B the measured cross-correlation amplitude $G_{\text {cross }}(0)$ was normalized (yielding $\left.G_{\text {cross }}^{\text {norm }}(0)\right)$ and plotted against the concentration of K4-Atto-655 in the bilayer (see SI for details). Then, $G_{\text {cross }}^{\text {norm }}(0)=1$ should be obtained if all $\mathrm{K}_{4}$-Atto- 655 binds to $\mathrm{CP}_{4} \mathrm{E}_{4}-\mathrm{Atto}-488$ molecules. $G_{\text {cross }}^{\text {norm }}(0)$ in Figure 3B grows with increasing concentration of K4-Atto-655 until it reaches a maximum value of about $25 \%$. Thus, considerable fractions of $\mathrm{K}_{4}-A$ tto- 655 and $\mathrm{CP}_{4} \mathrm{E}_{4}-\mathrm{Atto}-488$ do not bind to each other in the lipid bilayer.

Similarly, diffusion of $\mathrm{E}_{4}$-Atto-655 in the presence of $\mathrm{CP}_{4} \mathrm{~K}_{4}$-Atto-488 significantly differed from the diffusion of $\mathrm{CP}_{4} \mathrm{~K}_{4}$-Atto-488 in in the presence of 1 mol\% of $\mathrm{E}_{4}$ (Table 1). Moreover, the diffusion of $\mathrm{E}_{4}-$ Atto- 655 in pure DOPC/DOPE/Chol (50/25/25 mol\%) bilayers was much faster than the previously reported diffusion of 
$\mathrm{K}_{4}$-Atto-655. Both observations point to a weaker interaction of peptide $\mathrm{E}_{4}$ with the bilayer. Interestingly, it follows from the FCCS analysis of $\mathrm{CP}_{4} \mathrm{~K}_{4}$-Atto- 488 and $\mathrm{E}_{4}$-Atto-655 (Figure $3 \mathrm{~B}$ ) that only $35 \%$ of the maximum value of $G_{\text {cross }}(0)$ were reached in this case. In summary, we show that membrane embedded $\mathrm{CP}_{4} \mathrm{E}_{4}$ and $\mathrm{CP}_{4} \mathrm{~K}_{4}$ do form hetero-coils with the complementary $\mathrm{K}_{4}$ and $\mathrm{E}_{4}$, but the fraction of stable hetero-coils is low. It appears that the direct interaction of $E_{4}$ with $K_{4}$ is hampered by preferable interactions of $K_{4}$ with the bilayer. In the case when $\mathrm{K}_{4}$ binds to $\mathrm{CP}_{4} \mathrm{E}_{4}$ containing vesicles, $\mathrm{CP}_{4} \mathrm{E}_{4}$ works only as a handle which brings $\mathrm{K}_{4}$ close to the lipid bilayer where $\mathrm{K}_{4}$ prefers to interact with the bilayer rather than with $\mathrm{CP}_{4} \mathrm{E}_{4}$. In the other case when $\mathrm{E}_{4}$ is approaching $\mathrm{CP}_{4} \mathrm{~K}_{4}$-containing vesicles $\mathrm{E}_{4}$ can bind only by means of $\mathrm{CP}_{4} \mathrm{~K}_{4}$. This lipopeptide, however, is unable to bind $\mathrm{E}_{4}$ efficiently because of its high affinity to the bilayer.
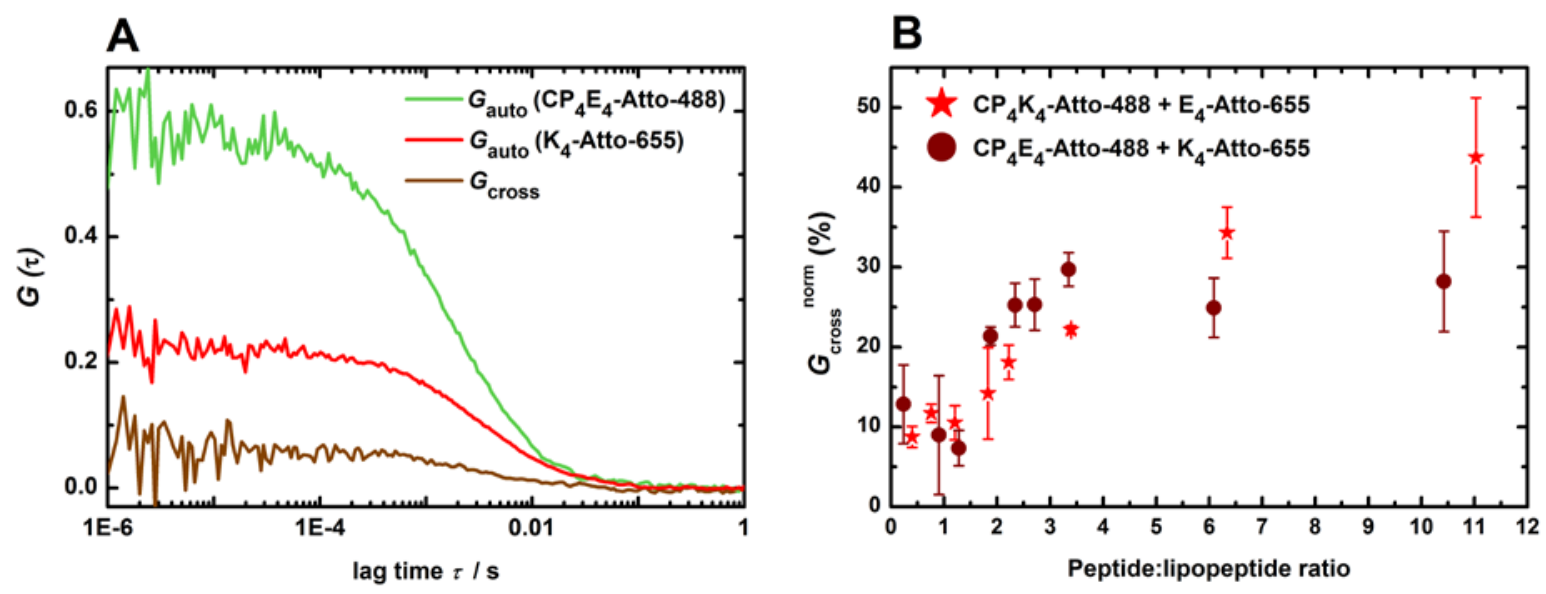

Figure 3. (Left): Representative auto-correlation functions $G_{\text {auto }}$ for $\mathrm{CP}_{4} \mathrm{E}_{4}-A t t o-488$ and $\mathrm{K}_{4}$-Atto- 655 obtained from an FCS measurement and the corresponding cross-correlation function ( $G_{\text {cross }}$ ) obtained from a parallel FCCS measurement. (Right): The normalized cross-correlation amplitude, which reports on the extent of interaction between one of the peptides and the complementary lipopeptide, as a function of the peptide to lipopeptide ratio on DOPC/DOPE/Chol (50/25/25 mol\%) bilayers. The average value of $G_{\text {cross }}^{\text {norm }}$ was calculated based on measurements on at least 5 GUVs.

Table 1. Diffusion coefficients $(D)$ together with their standard deviations for $\mathrm{K}_{4}$-Atto-655 and $\mathrm{E}_{4}-\mathrm{Atto}-655$ peptides adsorbed on DOPE/DOPC/Chol (50/25/25 mol\%) bilayers. The GUVs were pre-incubated with 1 mol\% of one of the (lipo)peptides specified in the first column. Furthermore, the table shows $D$ for a DOPEAtto-488 reference probe.

\begin{tabular}{|c|c|c|c|c|c|}
\hline \multirow{2}{*}{$\begin{array}{l}\text { GUVs pre-incubated } \\
\text { with } 1 \text { mol\% of }\end{array}$} & \multicolumn{5}{|c|}{$D\left(\mu \mathrm{m}^{2} / \mathrm{s}\right)$} \\
\hline & $\mathrm{K}_{4}$ Atto-655 & $E_{4}$-Atto-655 & $\mathrm{CP}_{4} \mathrm{~K}_{4}-$ Atto-488 & $\mathrm{CP}_{4} \mathrm{E}_{4}$-Atto-488 & DOPE-Atto-488 \\
\hline No LPs & $9.13 \pm 0.63$ & $132 \pm 13$ & - & - & $9.85 \pm 0.36$ \\
\hline $\mathrm{K}_{4}$ & - & - & - & $7.9 \pm 0.55$ & $9.73 \pm 0.54$ \\
\hline $\mathrm{CP}_{4} \mathrm{E}_{4}$ & $9.27 \pm 0.32$ & - & - & - & - \\
\hline $\mathrm{E}_{4}$ & - & - & $5.67 \pm 0.75$ & - & $10.09 \pm 0.58$ \\
\hline $\mathrm{CP}_{4} \mathrm{~K}_{4}$ & - & $10.24 \pm 0.95$ & - & - & - \\
\hline
\end{tabular}

Different distances of the peptide segments $E_{4}$ or $\mathrm{K}_{4}$ from the lipid water interface prevent lipopeptides from hetero-coiling

Importantly, no detectable hetero-coiling occurs when both lipopeptides $\mathrm{CP}_{n} \mathrm{E}_{4}$ and $\mathrm{CP}_{n} \mathrm{~K}_{4}$ are reconstituted in the same bilayer at roughly the same concentration. This is documented by the cross-correlation 
amplitude for $\mathrm{CP}_{4} \mathrm{E}_{4}$-Atto-488 and $\mathrm{CP}_{4} \mathrm{~K}_{4}$-Atto-655 equal to zero at low lipopeptide concentrations (<< 1 mol $\%, G_{\text {cross }}(0)=0$, Figure 4 A) but also by FRET at considerably higher lipopeptide concentrations (about 1 mol \%, Figure 4B), at which fusion normally progresses. The efficiency of FRET $E$ depends on the average distance of the donors from the acceptors. For the case of homogeneously distributed donors and acceptors in a lipid bilayer, the dependence of $E$ on the acceptor to lipid ratio can be obtained from a model derived by Baumann and Fayer (black line in Figure 4): it is steep at low acceptor to lipid ratios and flattens off typically at ratios exceeding 2 mol\% of the acceptors. ${ }^{20}$ Clustering of the donors with the acceptors (i.e. hetero-coiling of $\mathrm{CP}_{4} \mathrm{E}_{4}$-Atto-488 with $\mathrm{CP}_{4} \mathrm{~K}_{4}$-Atto-655) brings the donors effectively closer to the acceptors, enhancing the efficiency of FRET in comparison to the expected theoretical value for a given acceptor to lipid ratio. The hetero-coiling of lipopeptides should thus lead to a steeper dependence of $E$ on the acceptor to lipid ratio than would correspond to a homogeneous distribution of the labelled lipopeptides. The experimental dependency shown in Figure 4B perfectly follows the theoretical one (to understand in more detail how the theoretical dependence is calculated see $\mathrm{SI}$ ). This means that hetero-coils of the lipopeptides in the membrane are not formed at higher, and in terms of fusion more relevant, concentrations either (up to 1.2 mol\%). Such behaviour might suggest the two following scenarios: 1) hetero-coiling of lipopeptides is outcompeted by the formation of homo-coils or 2) the peptide segments of the complementary lipopeptides cannot come close enough to each other to facilitate hetero-coiling.

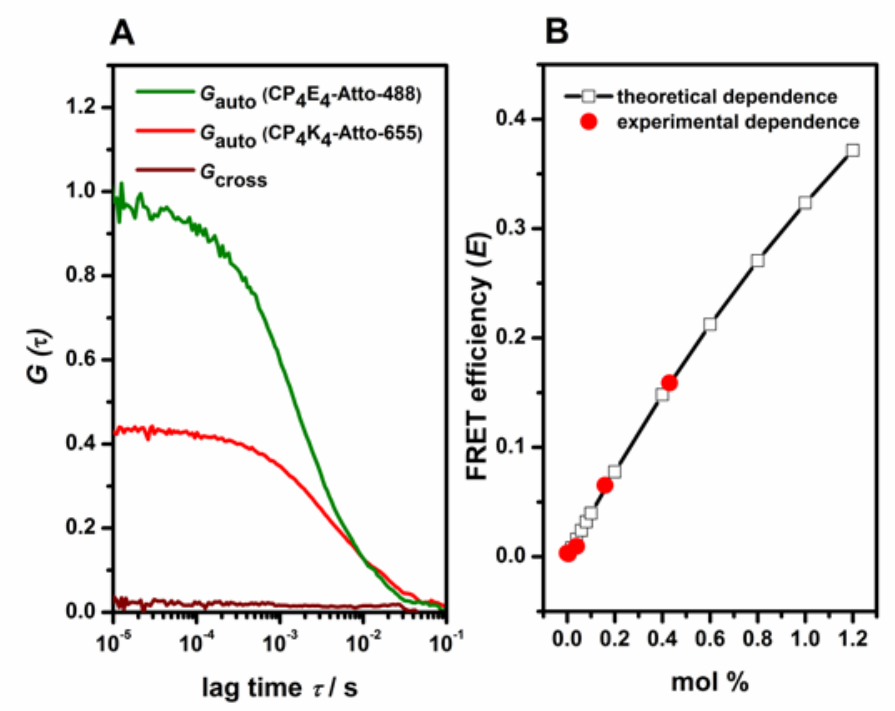

Figure 4. (A): Representative auto-correlation functions ( $G_{\text {auto }}$ ) for $\mathrm{CP}_{4} \mathrm{E}_{4}-\mathrm{Atto}-488$ and $\mathrm{CP}_{4} \mathrm{~K}_{4}-\mathrm{Atto}_{\mathrm{t}}-655$ and the corresponding cross-correlation function $\left(G_{\text {cross }}\right)$; $(B)$ : FRET efficiency $E$ as a function of the acceptor to lipid ratio. The donor/acceptor pair consisted of $\mathrm{CP}_{4} \mathrm{E}_{4}-A t t o-488 / \mathrm{CP}_{4} \mathrm{~K}_{4}-\mathrm{Atto}-655$ fluorescently labelled lipopeptides. The experimental data are displayed as solid red points. A theoretical dependence is shown for reference (black line with empty black squares). It was assumed in this dependence that the donors were homogeneously distributed in two parallel planes (separated by the distance $d_{1-1}\left(C_{4} \mathrm{E}_{4}-A_{t t o}-488\right)=d+2 d_{1}$. $\mathrm{m}\left(\mathrm{CP}_{4} \mathrm{E}_{4}\right.$-Atto-488)) whereas acceptors were distributed in two other parallel planes (separated by the distance $d_{1-1}\left(\mathrm{CP}_{4} \mathrm{~K}_{4}-\right.$ Atto-655) $=d+2 d_{1-\mathrm{m}}\left(\mathrm{CP}_{4} \mathrm{~K}_{4}-\right.$ Atto-655)). See Figure $\mathrm{S} 1$ for further explanation. The donor to lipid ratio was $0.3 \mathrm{~mol} \%$.

Scenario 1), i.e. homo-coiling of $\mathrm{CP}_{n} \mathrm{E}_{4}$ with $\mathrm{CP}_{n} \mathrm{E}_{4}$, or $\mathrm{CP}_{n} \mathrm{~K}_{4}$ with $\mathrm{CP}_{n} \mathrm{~K}_{4}$ has already been shown to impair membrane fusion. ${ }^{21}$ It has also been shown that aggregation of homo- and hetero-coils might enhance the efficiency of fusion or that it can induce membrane curvature and rupture, which might in turn mediate fusion. 2,16,17,19,22-24 However, neither our FCCS or brightness experiments conducted at low lipopeptide concentrations, nor FRET experiments performed at higher lipopeptide concentrations pointed to the presence of $\mathrm{CP}_{n} \mathrm{E}_{4}$ or $\mathrm{CP}_{n} \mathrm{~K}_{4}$ homo-coils (see SI for results). Despite an obvious absence of stable homo-coils in the DOPC/DOPE/Chol (50/25/25 mol\%) bilayer, individual lipopeptide molecules interfere with each other when present at fusion-relevant concentrations. This follows from the observation that after addition of 1 mol \% of the lipopeptides to the bilayer, the diffusion of a lipopeptide labelled by Atto-488 is slowed down 
more than the diffusion of a lipid-like reference probe DiD (1,1'-Dioctadecyl-3,3,3',3'Tetramethylindodicarbocyanine-5,5'-Disulfonic Acid, $D$ (lipopeptide)/D(DiD) $<1$, see the upper panel of Figure 5). It is likely that the relatively bulky headgroups of the lipopeptides consisting of a poly(ethylene glycol) chain and a peptide moiety $E_{4}$ or $K_{4}$ interfere with each other and cannot therefore move freely. According to Šachl et al., the diffusion under such conditions may still appear free although it is impeded, depending on the overall concentration of the obstacles. ${ }^{25}$ In other words, individual $\mathrm{CP}_{4} \mathrm{~K}_{4}$ or $\mathrm{CP}_{12} \mathrm{~K}_{4}$ molecules can act as obstacles for each other at such elevated concentrations.

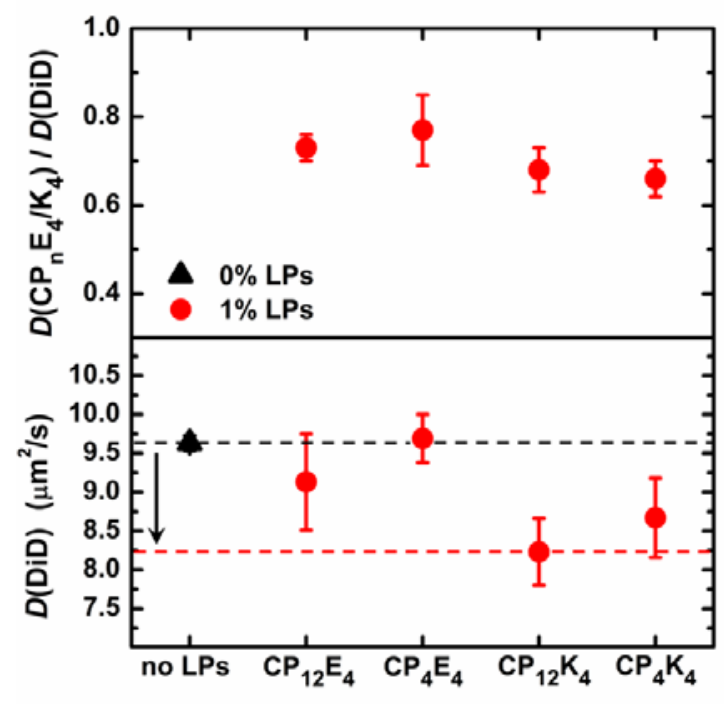

Figure 5. The diffusion coefficients $D$ of lipopeptides (LPs) $C P_{n} E_{4}$ or $C P_{n} K_{4}$ normalized by the diffusion coefficient of DiD (upper panel) and the diffusion coefficient of the membrane marker DiD (lower panel) shown for the lipopetide concentration of $1 \mathrm{~mol} \%$ in DOPC/DOPE/Chol (50/25/25 mol\%) GUVs.

Scenario 2) could happen if the positions of the peptide segments of the lipopeptides differed so much that the peptides would not encounter each other. This hypothesis can be tested by FRET. ${ }^{26}$ By assuming that the donors (peptide segments of $\mathrm{CP}_{n} \mathrm{E}_{4}-\mathrm{Atto}-488 / \mathrm{CP}_{n} \mathrm{~K}_{4}-\mathrm{Atto}-488$ ) were found in one plane whereas the acceptors (DiD) were localized at the lipid-water interface in the plane parallel to the first one, the distance between these planes could be determined (Figure 6). As shown in panel A of Figure 6 , the average timeresolved fluorescence decays of $\mathrm{CP}_{12} \mathrm{E}_{4}$-Atto-488 and $\mathrm{CP}_{12} \mathrm{~K}_{4}$-Atto-488 differ from each other significantly. Because the surface concentration of the acceptors was kept at a constant level the observed differences could have only been caused by different distances of the peptide segments from the interface. Fitting the decays with a model derived by Baumann and Fayer yielded quantitative information regarding the distances. ${ }^{20}$ The distance $d_{\text {CPK-m }}$ of Atto- 488 attached to peptide $\mathrm{K}_{4}$ in $\mathrm{CP}_{n} \mathrm{~K}_{4}$ was found, on average, $2.2-2.3$ $\mathrm{nm}$ from the acceptor plane (= the lipid-water interface). The estimated maximal theoretical distances of Atto-488 from this plane were calculated to be $6.5 \mathrm{~nm}$ for $\mathrm{CP}_{4} \mathrm{~K}_{4}$ and $9.3 \mathrm{~nm}$ for $\mathrm{CP}_{12} \mathrm{~K}_{4}$, respectively. Comparison of these values with the measured distance suggests 'looping back' of the peptide segments in $\mathrm{CP}_{4} \mathrm{~K}_{4}$ and $\mathrm{CP}_{12} \mathrm{~K}_{4}$ to the lipid-water interface. This finding is in line with previous findings, which reported the tendency of peptide $\mathrm{K}_{3}$ and the peptide segment $\mathrm{CP}_{n} \mathrm{~K}_{3}$ to snorkel in the lipid bilayer. ${ }^{17,19}$ Conversely, the distance $d_{\mathrm{CPE}-\mathrm{m}}$ of Atto-488 attached to $\mathrm{CP}_{n} \mathrm{E}_{4}$ from the bilayer surface equalled on average $(6.7 \pm 0.6) \mathrm{nm}$ for $\mathrm{CP}_{4} \mathrm{E}_{4} \mathrm{~nm}$ or $(6.0 \pm 0.5) \mathrm{nm}$ for $\mathrm{CP}_{12} \mathrm{E}_{4}$, respectively (Figure 6D). This means that $\mathrm{CP}_{n} \mathrm{E}_{4}$ is in contrast to $\mathrm{CP}_{n} \mathrm{~K}_{4}$ largely exposed to the bulk. Furthermore, as indicated by different time-resolved fluorescence decays obtained from individual GUVs (Figure 6B and S5) the peptide segments of both lipopeptides are broadly distributed around the corresponding average distances from the lipid-water interface. In summary, $\mathrm{E}_{4}$ in $\mathrm{CP}_{n} \mathrm{E}_{4}$, which is found more apart from the bilayer, has practically no chance to interact with $\mathrm{K}_{4}$ in $\mathrm{CP}_{n} \mathrm{~K}_{4}$, which is in contrast localized close to the lipid-water interface. Hetero-coils can apparently be formed only when $\mathrm{CP}_{n} \mathrm{E}_{4}$ and $\mathrm{CP}_{n} \mathrm{~K}_{4}$ approach each other as constituents of opposing membranes. 


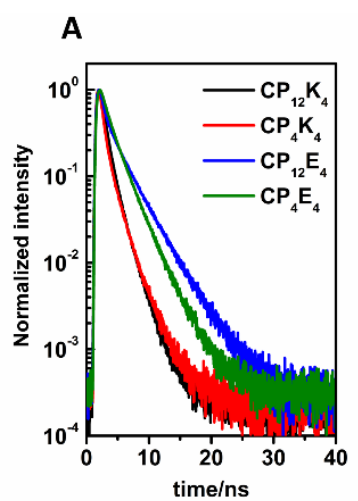

B
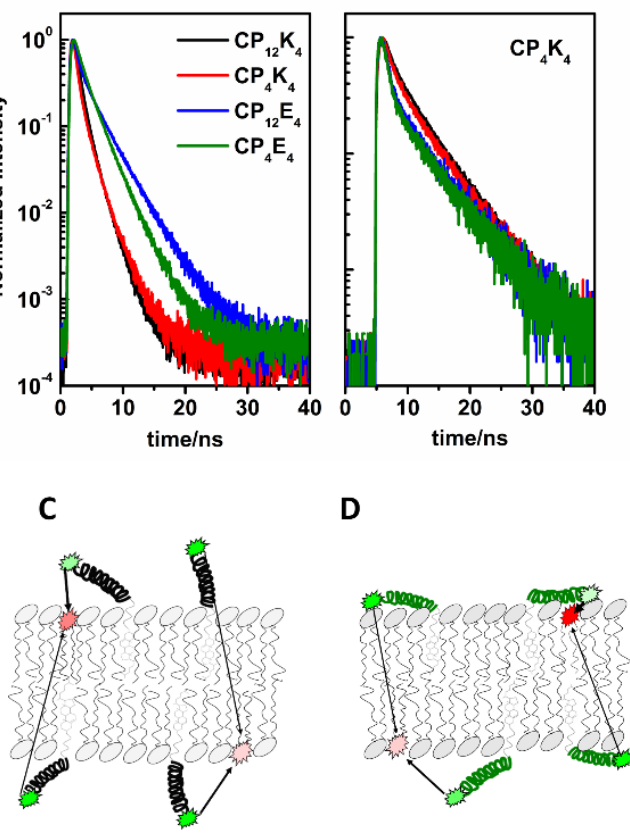

D

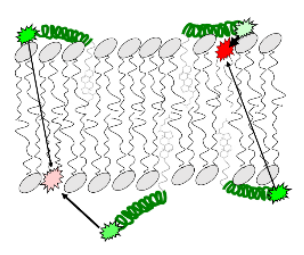

Figure 6. (A) Average normalized time-resolved fluorescence decays of $\mathrm{CP}_{n} \mathrm{E}_{4}-\mathrm{Atto}_{\mathrm{t}}-488$ and $\mathrm{CP}_{n} \mathrm{~K}_{4}-\mathrm{Atto}-488$ donors in the presence of DiD acceptors. (B) An example of varying decays of $\mathrm{CP}_{4} \mathrm{~K}_{4}-A t t o-488$ in the presence of DiD obtained for a set of different GUVs. (C-D) Schematic pictures outlying the proposed orientations of $\mathrm{CP}_{n} \mathrm{~K}_{4}(\mathrm{C})$ and $\mathrm{CP}_{n} \mathrm{E}_{4}$ (D) with respect to the lipid bilayer. The arrows point to the directions in which FRET can occur: within the same leaflet as well as across the lipid bilayer.

\section{Peptide $\mathrm{K}$ as an essential modulator of the lipid bilayer}

The different distances of the peptide segments of $\mathrm{CP}_{n} \mathrm{E}_{4}$ and $\mathrm{CP}_{n} \mathrm{~K}_{4}$ from the membrane strongly suggest that $\mathrm{K}_{4}$ will influence the DOPC/DOPE/Chol (50/25/25 mol\%) bilayer more than $\mathrm{E}_{4}$. Disruptive behaviour has already been reported for the lipopeptide $\mathrm{CP}_{n} \mathrm{~K}_{3}$ and peptide $\mathrm{K}_{3}$, which are shorter peptides. It was shown that $K_{3}$ reorganizes the membrane composition in its vicinity, induces positive membrane curvature, and enhances the probability of lipid tail protrusions. ${ }^{17}$ All of these effects are fusion relevant. ${ }^{27}$ Here we show by diffusion and Time-Dependent Fluorescence Shift (TDFS) measurements that $\mathrm{CP}_{4} \mathrm{~K}_{4}$ and $\mathrm{CP}_{12} \mathrm{~K}_{4}$ in contrast to $\mathrm{CP}_{4} \mathrm{E}_{4}$ and $\mathrm{CP}_{12} \mathrm{E}_{4}$ influence the bilayer at least to the level of carbonyls. TDFS provides information about hydration and mobility of the molecules that are found in the immediate vicinity of an excited probe. Both the hydration and mobility can be quantified by the total spectral shift $(\Delta v)$ and the mean solvent relaxation time $\left(\tau_{r}\right)$, respectively. It has been shown that, for Laurdan located at the fully hydrated carbonyl level of a phospholipid bilayer, $\Delta v$ is directly proportional to the hydration and $\tau_{r}$ to the rigidity of the lipid bilayer at the level of the carbonyls. ${ }^{28,29}$ As follows from Figure 7, 2 mol\% of $\mathrm{CP}_{4} \mathrm{E}_{4}$ and $\mathrm{CP}_{12} \mathrm{E}_{4}$ were not able to induce any significant changes in hydration or mobility; and as follows from the lower panel of Figure 5, the diffusion of a fluorescent lipid analog DiD remained unaffected by addition of 1 mol \% of $\mathrm{CP}_{4} \mathrm{E}_{4}$ or $\mathrm{CP}_{12} \mathrm{E}_{4}$. On the other hand, addition of 2 mol\% of both $\mathrm{CP}_{4} \mathrm{~K}_{4}$ and $\mathrm{CP}_{12} \mathrm{~K}_{4}$ resulted in the prolongation of $\tau_{\mathrm{r}}$ between 11 and $15 \%$, a decrease in $\Delta v$ from 4350 to $4250 \mathrm{~cm}^{-1}$ and an impeded diffusion of DiD (Figure 5 and 6). Prolongation of $\tau_{r}$ is usually accompanied in TDFS experiments by the decrease in $\Delta v$ because of the increase in bilayer rigidity, mostly caused by denser lipid packing, and this often leads to dehydration as water molecules are expelled to the bulk. ${ }^{30-32}$ In summary, $\mathrm{CP}_{4} \mathrm{~K}_{4}$ and $\mathrm{CP}_{12} \mathrm{~K}_{4}$ do affect the bilayer by increasing bilayer viscosity and by dehydrating the bilayer down to the carbonyl level. 


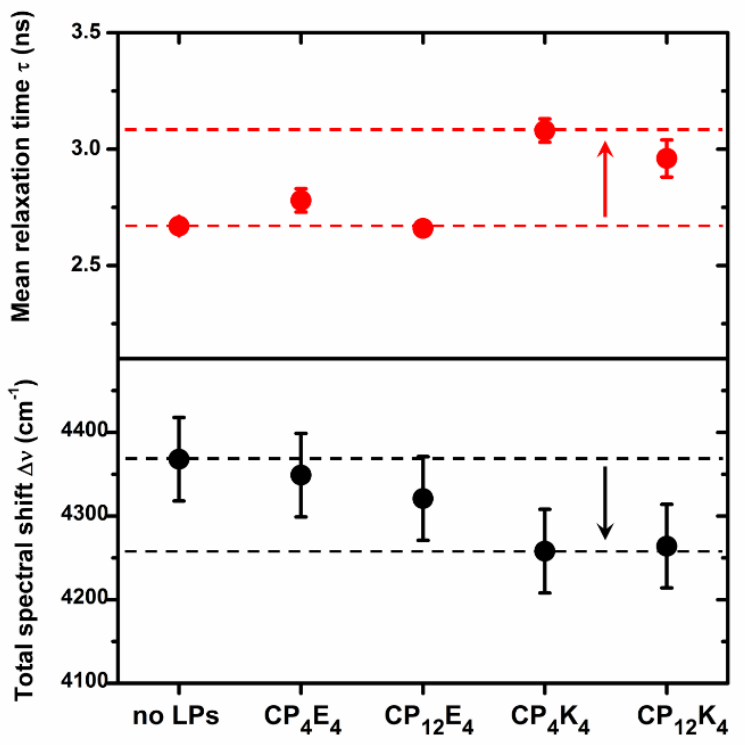

Figure 7. The solvent relaxation time $\tau_{r}$ and the spectral shift $\Delta v$ of Laurdan in DOPC/DOPE/Chol (50/25/25 mol\%) LUVs containing 2 mol\% of lipopeptides (LPs).

\section{Molecular view of the initial steps of membrane fusion}

It is generaly accepted nowadays that membrane fusion requires such molecules that bring the opposing membranes into close proximity and locally disturb the lipid bilayers in order to reduce the energetic costs of membrane fusion. This work should prove that fusion caused by lipopeptides $\mathrm{CP}_{n} \mathrm{E}_{4}$ and $\mathrm{CP}_{n} \mathrm{~K}_{4}$ also proceeds according to this general scheme.

The fusion driven by $\mathrm{CP}_{n} \mathrm{E}_{4}$ and $\mathrm{CPnK}_{4}$ is based on coiled-coil formation between peptides $\mathrm{E}_{4}$ and $\mathrm{K}_{4}$. The formation of this coiled-coil is intended to mediate close contact of opposing membranes that the peptides are anchored in. We have already shown that $\mathrm{K}_{4}$, in contrast to $\mathrm{E}_{4}$, interacts strongly with DOPC/DOPE/Chol (50/25/25 mol\%) bilayers. Such strong binding is probably facilitated by the specific positioning of lysine residues and respective charge distribution within the primary sequence of peptide $\mathrm{K}_{4} \cdot{ }^{19}$ Amphipathic helices with this charge distribution pattern are classified as class A1 amphipathic helices and are known to interact with zwitterionic lipids. ${ }^{33,34}$ Both $\mathrm{CP}_{n} \mathrm{E}_{4}$ and $\mathrm{CP}_{n} \mathrm{~K}_{4}$ have been found in DOPC/DOPE/Chol (50/25/25 mol\%) bilayers as monomers. Neither FCCS nor FRET experiments revealed any detectable amount of stable homo-oligomers in DOPC/DOPE/Chol (50/25/25 mol\%) bilayers at a broad range of lipopeptide concentrations $0.05-1.2$ mol\%. Such oligomers might potentially reduce the number of monomeric peptides which are available for binding to a complementary lipopeptide. Formation of homo-oligomers was suggested by previous studies employing circular dichroism spectroscopy. Increased $\alpha$-helicity, commonly attributed to the homo-coil formation, was shown for peptides in solution and for lipopeptides incorporated in model lipid bilayers at concentration spanning the range at which the fusion normally occurs (1-3 mol\%). ${ }^{12,13,18,35-37}$ According to a recent study, elevated $\alpha$-helicity of $\mathrm{CP}_{12} \mathrm{~K}_{3}$ does not reflect the peptide homo-coiling, but rather membrane snorkelling of $\mathrm{K}_{3}$ moiety into the lipid bilayer. ${ }^{16}$ In contrast to $\mathrm{CP}_{12} \mathrm{~K}_{3}$, no such interaction was shown for $\mathrm{CP}_{12} \mathrm{E}_{3}$, which was reported to exist in equilibrium between unfolded monomers and folded homo-coils at 2 mol\%. Although we could not reveal any stable homo-clusters below 1.2 mol\% by our methods, it follows from FCS diffusion measurements that the lipopeptides interfere with each other because the diffusion of all lipopeptides is slowed down just because of the presence of the lipopeptides more than the diffusion of DiD. Interactions of $\mathrm{CP}_{n} \mathrm{E}_{4}$ with $\mathrm{CP}_{n} \mathrm{~K}_{4}$ are happening in a dense matrix of neighbouring lipopeptides. Such a dense environment of surrounding lipopeptides might modulate homo- and hetero-coiling of $\mathrm{CP}_{n} \mathrm{E}_{4}$ and $\mathrm{CP}_{n} \mathrm{~K}_{4}$.

Importantly, binding of $\mathrm{K}_{4}$ to $\mathrm{E}_{4}$ is not efficient. According to single molecule FCCS experiments, the majority of $\mathrm{CP}_{4} \mathrm{E}_{4}$ and $\mathrm{K}_{4}$ remain unbound to each other when up to a 10 -fold excess of $\mathrm{K}_{4}$ is added to $C \mathrm{P}_{4} \mathrm{E}_{4}$ containing 
GUVs. Of note, the cross-correlation amplitude reaches about $30 \%$ of the maximum value that could be reached. Furthermore, binding of $\mathrm{K}_{4}$ to $\mathrm{CP}_{n} \mathrm{E}_{4}$ rich GUVs is 6 times stronger than to $\mathrm{CP}_{n} \mathrm{E}_{4}$ deficient GUVs, and as indicated by diffusion measurements, $\mathrm{K}_{4}$ sticks to the bilayer, where it diffuses with almost the same speed as the surrounding lipids. Therefore, our FCCS experiments directly prove the exclusive function of $\mathrm{CP}_{n} \mathrm{E}_{4}$, which is to recruit peptide $\mathrm{K}_{4}$ to the bilayer. Similarly, it appears from FCCS experiments that significant amounts of $\mathrm{CP}_{4} \mathrm{~K}_{4}$ and $\mathrm{E}_{4}$ stay unbound to each other when up to a 10-fold excess of $\mathrm{E}_{4}$ is incubated with $\mathrm{CP}_{4} \mathrm{~K}_{4}$ containing GUVs. The cross-correlation amplitude is even lower in this case as compared to the previous one. This occurs because the equilibrium between free $\mathrm{CP}_{4} \mathrm{~K}_{4}$ and $\mathrm{E}_{4}$ and the resulting hetero-coil is shifted in favour of the free components and is overwhelmed by interactions between $\mathrm{CP}_{4} \mathrm{~K}_{4}$ and the lipid bilayer. $\mathrm{E}_{4}$ behaves differently in comparison to $\mathrm{K}_{4}$ in that it interacts with the bilayer exclusively by means of the complementary lipopeptide $\mathrm{CP}_{n} \mathrm{~K}_{4}$. Therefore, the function of $\mathrm{CP}_{n} \mathrm{~K}_{4}$ is actually two-fold: firstly it brings the fusing bilayers into close contact; and secondly it strongly interacts with the lipid bilayer.

Surprisingly, $\mathrm{CP}_{n} \mathrm{E}_{4}$ and $\mathrm{CP}_{n} \mathrm{~K}_{4}$ do not form hetero-coils so efficiently with each other when reconstituted in the same bilayer. The efficiency of hetero-coiling is in fact so low that FCCS was not able to reveal any detectable amount of $\mathrm{CP}_{4} \mathrm{E}_{4}$-Atto-488/CP4 $\mathrm{K}_{4}$-Atto-655 pairs (see above). This can be rationalized by the fact that $\mathrm{CP}_{n} \mathrm{~K}_{4}$ and $\mathrm{CP}_{n} \mathrm{E}_{4}$ are localized at different distances from the lipid-water interface with a low chance of meeting/interacting with each other. The different localization of the peptides along the bilayer normal may represent an important mechanism by which the number of free $\mathrm{CP}_{n} \mathrm{E}_{4}$ and $\mathrm{CP}_{n} \mathrm{~K}_{4}$ molecules potentially available for binding to an opposing membrane is kept on a high level, enabling efficient fusion. Furthermore, it follows from the FRET experiments we performed that the transverse distribution of $\mathrm{K}_{4}$ and $\mathrm{E}_{4}$ peptide moiety of $\mathrm{CP}_{n} \mathrm{~K}_{4} / \mathrm{CP}_{n} \mathrm{E}_{4}$ molecules is rather broad. Whereas the peptide segment of $\mathrm{CP}_{n} \mathrm{E}_{4}$ is exposed to the bulk, a considerable fraction of $\mathrm{CP}_{n} \mathrm{~K}_{4}$ is because of the broad transverse distribution imbedded in the bilayer. Previous experiments showed that fusion of $\mathrm{CP}_{n} \mathrm{E}_{3}$ or $\mathrm{CP}_{n} \mathrm{~K}_{3}$ containing LUVs occurs only if the length of the poly(ethylene glycol) linker of $\mathrm{CP}_{n} \mathrm{E}_{3}$ is so long that it can reach the lipid-water interface of the opposing bilayer where majority of $\mathrm{CP}_{\mathrm{n}} \mathrm{K}_{4}$ is imbedded. ${ }^{38}$ Therefore, it appears crucial for the fusion that the peptide segment of $\mathrm{CP}_{n} \mathrm{E}_{4}$ is sufficiently long and exposed to the bulk. The bilayers, which do not carry any electric charge, can approach each other up to the equilibrium distance of 2-3 $\mathrm{nm}$. In this respect, the distance equal to about $6 \mathrm{~nm}$ of Atto-488 attached to $\mathrm{CP}_{n} \mathrm{E}_{4}$ from the bilayer surface seems optimal for the fusion to occur.

Lipopeptides $\mathrm{CP}_{n} \mathrm{E}_{4}$ in contrast to $\mathrm{CP}_{n} \mathrm{~K}_{4}$ do not have any observable impact on DOPC/DOPE/Chol (50/25/25 mol\%) bilayers. The peptide segments $\mathrm{K}_{4}$ of $\mathrm{CP}_{n} \mathrm{~K}_{4}$ molecules densely cover the surface of the bilayer at 2 mol\% of $\mathrm{CP}_{n} \mathrm{~K}_{4}$, which leads to increased microviscosity and decreased polarity of the carbonyl region of the lipid bilayer. Importantly, dehydration of the lipid bilayer was shown to play an important role during membrane fusion. ${ }^{39,40}$ Moreover, the diffusion of the lipid analogue DiD becomes impeded by 1 mol\% of $\mathrm{CP}_{n} \mathrm{~K}_{4}$. These findings are in line with a peptide insertion model with the helical peptide inserted in parallel to the membrane surface and with the hydrophobic face penetrating into the hydrophobic core of the bilayer. ${ }^{17}$ From the macroscopic point of view, the insertion of $\mathrm{K}_{4}$ was reported to be accompanied by local membrane deformations, which are manifested by an altered bilayer curvature and lipid tail protrusions. These initial steps of membrane fusion are followed by fusion of the proximal leaflets and lipid mixing culminated by opening of a fusion pore and content mixing. However, how these later stages of membrane fusion occur still remains a question.

\section{Lipopeptide versus SNARE mediated membrane fusion}

The fusion system based on $\mathrm{CP}_{n} \mathrm{E}_{4}$ and $\mathrm{CP}_{n} \mathrm{~K}_{4}$ is a reduced system derived from SNARE proteins which constitute central molecules in the fusion machinery of eukaryotic cells. ${ }^{12}$ This implies that the fusion mechanisms for both the reduced and original systems do not need to be fully identical. Both mechanisms are driven by self-association of alpha-helixes containing several heptad repeat sequences with high propensity to form coiled coils. ${ }^{41,12}$ Nevertheless, whereas the lipopeptide mediated fusion requires only two complementary molecules $\mathrm{CP}_{n} E_{4}$ and $\mathrm{CP}_{n} \mathrm{~K}_{4}$, the SNARE mediated fusion is based on self-association of at least one v-SNARE (synaptobrevin) and two t-SNARE (syntaxin and SNAP-25) proteins. ${ }^{41}$ In both cases, the complex formation is accompanied by energy release which is used to bring the opposing membranes into 
close proximity ${ }^{42}$ Critical steps in membrane fusion represent dehydration of the lipid bilayer and formation of hydrophobic contacts between the approaching bilayers. ${ }^{6}$ These contacts are formed by hydrophobic acyl chains protruding to the lipid-water interface. ${ }^{39}$ There is a good agreement that both the lipopeptides as well as the SNAREs are able to increase the hydrophobicity of the lipid-water interface. ${ }^{17,41}$ However, whereas in case of the lipopeptides it is only $\mathrm{CP}_{n} \mathrm{~K}_{4}$ that is able to dehydrate the lipid bilayer, in case of SNAREs dehydration has so far been experimentally demonstrated only for synaptobrevin or for the whole SNARE complex. ${ }^{43,44}$

A fundamental difference between both mechanisms might lie in the fact that other parts of a SNARE molecule may as well contribute to the fusion. These regions include the transmembrane domain, which anchors the protein in the membrane, and the linker region, which connects the transmembrane domain with the bulk alpha helical repeats. Reports have appeared that the linker region is able to transmit the stress induced by self-assembly of SNAREs into the membrane and to push the transition from hemi to full fusion by its positively charged residues..$^{45,46,42}$ The transmembrane domain, on the other hand, should be critical for accomplishing the fusion by opening a fusion pore ${ }^{47}$ It could also be responsible for homo- and hetero-oligomerization of SNAREs, which would explain why homo-oligomers of SNAREs are formed more efficiently than those formed by the lipopeptides. ${ }^{48}$ The system based on the lipopeptides represents the most simplified system where fusion still occurs. From this perspective it seems that the transmembrane domain and the linker region of SNAREs have rather a regulatory than an indispensable function. This view has recently been supported by fusion experiments performed on lipid anchored SNAREs during neurotransmitter release, but it will require further supportive experiments on other systems, too. ${ }^{49}$

\section{Conclusions}

Fusion of intracellular membranes in nature is mediated by so-called SNARE proteins. A minimal model system for membrane fusion inspired by these proteins consists of cholesterol serving as a lipid membrane anchor, a poly(ethylene glycol) linker and either a cationic peptide $\mathrm{K}_{4}$ or its counterpart an anionic peptide $E_{4}$. The behaviour of the complementary lipopeptides $\mathrm{CP}_{n} \mathrm{E}_{4}$ and $\mathrm{CP}_{n} \mathrm{~K}_{4}$ is in many ways different, which fits with their previously uncovered distinct roles during fusion. The lipopeptide molecules $\mathrm{CP}_{n} \mathrm{~K}_{4}$ exist in lipid bilayers predominantly as monomers where they strongly interact with the lipid bilayer. During the initial steps of membrane fusion, the main role of $\mathrm{CP}_{n} \mathrm{~K}_{4}$ is to disrupt the bilayer and poise it for undergoing fusion. On the other hand, $\mathrm{CP}_{\mathrm{n}} \mathrm{E}_{4}$ molecules work as lipid anchors. The peptide moieties are exposed to the bulk, where they search for the complementary $\mathrm{CP}_{n} \mathrm{~K}_{4}$ molecules, recruiting them to their own bilayer. The efficiency of hetero-coil formation is very low and possible only when the lipopeptides approach each other as constituents of different bilayers. When the complementary lipopeptides are incorporated in the same bilayer the formation of hetero-coils is disabled by different localization of the peptides along the normal of the lipid bilayer. This mechanism keeps the number of monomeric lipopeptides that can hetero-coil with a complementary lipopeptide on the neighbouring bilayer at a high level, enabling efficient fusion. All these facts represent important findings that need to be taken into account when a model for later stages of fusion is developed.

\section{Methods}

General. Details of all chemicals, synthesis of the (lipo)peptides and formation of GUVs can be found in the supporting information.

Sample preparation for FCS (fluorescence correlation spectroscopy) and FCCS (fluorescence cross-correlation spectroscopy). The lipopeptides dissolved in methanol were added to the GUVs by keeping the volume of added solvent below $1 \%$ of the total volume. Prior to a measurement, the GUVs were incubated with the lipopeptides for at least 30 minutes. Finally, $40 \mu \mathrm{l}$ of GUVs were added to a microscope chamber (Nunc ${ }^{\circ}$ Lab-Tek $^{\circ}$ Chamber) filled with $360 \mu$ of phosphate buffer ( $25 \mathrm{mM}$ PBS, $100 \mathrm{mM} \mathrm{KCl}, 1 \mathrm{mM}$ EDTA, pH 7.4, 255 mOsm) and precoated with BSA-biotin/streptavidin for immobilization of the GUVs at the bottom of the microscope chamber. The probe to lipid ratio was between $0.1 \%$ and $0.005 \%$. The bilayer of GUVs contained in addition a lipophilic marker DiD at $0.001 \mathrm{~mol} \%$. 
Sample preparation for FLIM-FRET (fluorescence lifetime imaging of Förster resonance energy transfer). The preparation procedure was similar to that one described in the previous section except that the lipopeptides were mixed with the lipids already before the formation of GUVs. $40 \mu$ l of GUVs were added to a microscope chamber (Nunc ${ }^{\circ}$ Lab-Tek $^{\circ}$ Chamber), filled with $360 \mu$ l of phosphate buffer $(25 \mathrm{mM}$ PBS, $100 \mathrm{mM} \mathrm{KCl}, 1 \mathrm{mM}$ EDTA, pH 7.4, $255 \mathrm{mOsm}$ ) and precoated with BSA-biotin/streptavidin. Prior to a measurement, the GUVs were left for about $15 \mathrm{~min}$ to settle down at the bottom of a chamber.

Sample preparation for Time-Dependent Fluorescence Shifts (TDFS) of Laurdan. Appropriate volumes of lipids dissolved in $\mathrm{CHCl}_{3}$ and Laurdan dissolved in MetOH were mixed in glass tubes and dried under nitrogen stream. To get rid of the remaining organic solvents the lipid films were left in vacuum for at least two hours. The resulting lipid films were resuspended in phosphate buffer $(25 \mathrm{mM}$ PBS, $100 \mathrm{mM} \mathrm{KCl}, 1 \mathrm{mM}$ EDTA, pH 7.4). Large unilamellar vesicles (LUVs) were formed by extrusion through filters with a defined pore size of $100 \mathrm{~nm}$ (Avestin, Ottawa, Canada). Final concentrations of lipids and Laurdan were $1 \mathrm{mM}$ and $0.01 \mathrm{mM}$, respectively. The samples were equilibrated for at least 15 minutes prior to a measurement.

FLIM-FRET, FCS and FCCS measurements were performed on a home build confocal microscope consisting of an inverted confocal microscope body IX71 (Olympus, Hamburg, Germany). The samples were excited by pulsed diode lasers (LDH-P-C-470, $\lambda=470 \mathrm{~nm}$ and LDH-D-C-635, $\lambda=635 \mathrm{~nm}$, both produced by Picoquant, Germany) with the repetition rate of $12.5 \mathrm{MHz}$ for each of the laser lines. The laser light was pulsed alternatively to avoid artefacts caused by signal bleed-through. The light was up-reflected to a water immersion objective (UPLSAPO 60x, Olympus) with a 470/635 nm dichroic mirror. The signal was detected by two single photon avalanche diode detectors equipped with $515 / 50$ and $685 / 50 \mathrm{~nm}$ band pass filters (Chroma Rockingham, VT).

Z-scans were conducted at the top of a single GUV. The membrane was scanned vertically in 15 steps separated $150 \mathrm{~nm}$ apart from each other. 60-seconds-long intensity trace was recorded at each position. To obtain the average diffusion coefficients measurements on at least five different GUVs were done. Further details on the analysis of data can be found for instance in ${ }^{50}$. During acquisition of FLIM-FRET data, GUVs were scanned at the cross-section with the resolution of $512 \times 512$ pixels $(0.6 \mathrm{~ms} / \mathrm{pixel})$. Decays from at least five different GUVs were summed up and used for further analysis. The temperature was kept at $25^{\circ} \mathrm{C}$. The analysis of FLIM-FRET, FCS and FCCS is described in more detail in supporting information (SI) of this paper.

TDFS measurements. Steady-state emission spectra were measured on Fluorolog-3 spectrofluorometer (model FL3-11; Jobin Yvon Inc., Edison, NJ, USA) equipped with a xenon-arc lamp whereas time-resolved fluorescent decays were recorded using a 5000U Single Photon Counting setup equipped with a cooled Hamamatsu R3809U-50 microchannel plate photomultiplier (IBH, UK). Laurdan was excited at $373 \mathrm{~nm}$ with an IBH NanoLed 11 laser diode. The data were collected for a series of wavelengths ranging from $400 \mathrm{~nm}$ to $540 \mathrm{~nm}$ with a $10 \mathrm{~nm}$ step. Potentially present scattered light was eliminated by a cut-off emission filter > $399 \mathrm{~nm}$. Each decay was fitted with a multi-exponential function using the iterative reconvolution procedure (IBH DAS6 software). Time-resolved emission spectra (TRES) were reconstructed from the recorded series of fluorescent decays and the corresponding steady-state emission spectrum. ${ }^{51}$ To determine positions of maxima in TRES, $v(t)$, the TRES spectra were fitted by a log-normal function. The total spectral shift $\Delta v$ was calculated as $\Delta v=[v(0)-v(\infty)]$. The so-called correlation function of solvent relaxation is expressed as $C(t)=[v(t)-v(\infty)] /[\Delta v]$ and allows for quantitative description of solvation dynamics occurring in the system. Finally, the mean solvent relaxation time equals $\tau_{r}=\int_{0}^{\infty} C(t) \mathrm{d} t$ per definition. TDFS experiments were performed at $283 \mathrm{~K}$.

\section{Conflicts of interest}

There are no conflicts to declare.

\section{Acknowledgements}

RŠ acknowledges financial support from the Czech Science Foundation via grant 18-04871S. AK:á acknowledges the support of the Czech Science Foundation (17-05903S). AK:s and ALB acknowledge the 
support of the Netherlands Organization for Scientific Research (NWO), via a VICl grant (724.014.001) to AK:s and a VENI grant (722.015.006) to ALB.

\section{Notes and references}

R. Jahn, T. Lang and T. C. Südhof, Cell, 2003, 112, 519-533.

S. Martens and H. T. McMahon, Nat. Rev. Mol. Cell Biol., 2008, 9, 543-56.

X. Chen, D. Araç, T.-M. Wang, C. J. Gilpin, J. Zimmerberg and J. Rizo, Biophys. J., 2006, 90, 2062-74.

H. Robson Marsden and A. Kros, Angew. Chem. Int. Ed. Engl., 2010, 49, 2988-3005.

T. C. Südhof and J. E. Rothman, Science, 2009, 323, 474-7.

L. V Chernomordik, G. B. Melikyan and Y. A. Chizmadzhev, Biochim. Biophys. Acta, 1987, 906, 309-52.

M. M. Kozlov and V. S. Markin, Biofizika, 28, 242-7.

G. Stengel, R. Zahn and F. Höök, J. Am. Chem. Soc., 2007, 129, 9584-5.

A. Kashiwada, M. Tsuboi and K. Matsuda, Chem. Commun. (Camb)., 2009, 695-7.

M. Ma, A. Paredes and D. Bong, J. Am. Chem. Soc., 2008, 130, 14456-8.

Y. Gong, Y. Luo and D. Bong, J. Am. Chem. Soc., 2006, 128, 14430-1.

H. Robson Marsden, N. A. Elbers, P. H. H. Bomans, N. A. J. M. Sommerdijk and A. Kros, Angew. Chem. Int. Ed. Engl., 2009, 48, 2330-3.

F. Versluis, J. Voskuhl, B. van Kolck, H. Zope, M. Bremmer, T. Albregtse and A. Kros, J. Am. Chem. Soc., 2013, 135, 80578062.

H. R. Zope, F. Versluis, A. Ordas, J. Voskuhl, H. P. Spaink and A. Kros, Angew. Chem. Int. Ed. Engl., 2013, 52, $14247-51$. N. L. Mora, A. Bahreman, H. Valkenier, H. Li, T. H. Sharp, D. N. Sheppard, A. P. Davis and A. Kros, Chem. Sci., 2016, 7, 1768-1772.

M. Rabe, H. R. Zope and A. Kros, Langmuir, 2015, 31, 9953-9964.

M. Rabe, C. Aisenbrey, K. Pluhackova, V. de Wert, A. L. Boyle, D. F. Bruggeman, S. A. Kirsch, R. A. Böckmann, A. Kros, J. Raap and B. Bechinger, Biophys. J., 2016, 111, 2162-2175.

H. Robson Marsden, A. V. Korobko, T. Zheng, J. Voskuhl and A. Kros, Biomater. Sci., 2013, 1, 1046.

M. Rabe, C. Schwieger, H. R. Zope, F. Versluis and A. Kros, Langmuir, 2014, 30, 7724-7735.

J. Baumann and M. D. Fayer, J. Chem. Phys., 1986, 85, 4087.

G. Pähler, B. Lorenz and A. Janshoff, Biochem. Biophys. Res. Commun., 2013, 430, 938-43.

Y. A. Chen and R. H. Scheller, Nat. Rev. Mol. Cell Biol., 2001, 2, 98-106.

L. Shi, Q.-T. Shen, A. Kiel, J. Wang, H.-W. Wang, T. J. Melia, J. E. Rothman and F. Pincet, Science (80-. )., 2012, 335, 1355-1359.

R. Mohrmann, H. de Wit, M. Verhage, E. Neher and J. B. Sorensen, Science (80-. )., 2010, 330, 502-505.

R. Šachl, J. Bergstrand, J. Widengren and M. Hof, J. Phys.D Appl. Phys., 2016, 49, 114002 (11pp).

R. Šachl, I. Boldyrev and L. B.-Å. Johansson, Phys. Chem. Chem. Phys., 2010, 12, 6027-6034.

S. Y. Woo and H. Lee, Sci. Rep., 2016, 6, 1-13.

Š. Pokorná, A. Olżyńska, P. Jurkiewicz and M. Hof, Springer Berlin Heidelberg, 2012, pp. 141-159.

P. Jurkiewicz, J. Sýkora, A. Olzyńska, J. Humpolíčková and M. Hof, J. Fluoresc., 2005, 15, 883-894.

P. Jurkiewicz, L. Cwiklik, P. Jungwirth and M. Hof, Biochimie, 2012, 94, 26-32.

P. Jurkiewicz, L. Cwiklik, A. Vojtíšková, P. Jungwirth and M. Hof, Biochim. Biophys. Acta-Biomembr., 2012, 1818, 609616.

A. Melcrová, Š. Pokorná, S. Pullanchery, M. Kohagen, P. Jurkiewicz, M. Hof, P. Jungwirth, P. S. Cremer and L. Cwiklik, Sci. Rep., 2016, 6, 38035.

J. P. Segrest, H. De Loof, J. G. Dohlman, C. G. Brouillette and G. M. Anantharamaiah, Proteins Struct. Funct. Genet., 1990, 8, 103-117.

Vinod K. Mishra and M. N. Palgunachari, Biochemistry, 1996, 35, 11210-11220.

M. Rabe, A. Boyle, H. R. Zope, F. Versluis and A. Kros, Biopolymers, 2015, 104, 65-72.

T. Zheng, J. Voskuhl, F. Versluis, H. R. Zope, I. Tomatsu, H. R. Marsden, A. Kros, J. E. Rothman and X. Y. Zhu, Chem. Commun., 2013, 49, 3649.

I. Tomatsu, H. R. Marsden, M. Rabe, F. Versluis, T. Zheng, H. Zope and A. Kros, J. Mater. Chem., 2011, $21,18927$.

G. A. Daudey, H. R. Zope, J. Voskuhl, A. Kros and A. L. Boyle, Langmuir, 2017, 33, 12443-12452.

P. K. J. Kinnunen, Chem. Phys. Lipids, 1992, 63, 251-258.

J. M. Holopainen, J. Y. A. Lehtonen and P. K. J. Kinnunen, Biophys. J., 1999, 76, 2111-2120.

J. Han, K. Pluhackova and R. A. Bïckmann, Front. Physiol., , DOI:10.3389/fphys.2017.00005.

J. M. Hernandez, A. Stein, E. Behrmann, D. Riedel, A. Cypionka, Z. Farsi, P. J. Walla, S. Raunser and R. Jahn, Science (80-. )., 2012, 336, 1581-1584.

J. Han, K. Pluhackova, D. Bruns and R. A. Böckmann, Biochim. Biophys. Acta-Biomembr., 2016, 1858, 855-865. 
44

T. A. M. Bharat, J. Malsam, W. J. H. Hagen, A. Scheutzow, T. H. Söllner and J. A. G. Briggs, EMBO Rep., 2014, 15, 308-14. V. Knecht and H. Grubmüller, Biophys. J., 2003, 84, 1527-1547.

R. Jahn and D. Fasshauer, Nature, 2012, 490, 201-207.

‡ S. Moses Dennison, § Norma Greenfield, and John Lenard and ¥ Barry R. Lentz*, , DOI:10.1021/BI0203233.

X. Lu, Y. Zhang and Y.-K. Shin, Nat. Struct. Mol. Biol., 2008, 15, 700-706.

P. Zhou, T. Bacaj, X. Yang, Z. P. Pang and T. C. Südhof, Neuron, 2013, 80, 470-483.

A. Benda, M. Beneš, V. Mareček, A. Lhotský, W. T. Hermens and M. Hof, Langmuir, 2003, 19, 4120-4126.

M. L. G. Horng Ja Papazyan, a Maroncelli, M, J. Phys. Chem., 1995, 99, 17337. 